\title{
Unrecognized Interstitial Lung Disease as a Result of Chronic Nitrofurantoin Use
}

\author{
Kerry Anne Rambaran ${ }^{1} \cdot$ Charles F. Seifert $^{1}$
}

Published online: 24 October 2016

(C) The Author(s) 2016. This article is published with open access at Springerlink.com

\begin{abstract}
Drug-induced interstitial lung disease is a rare condition attributed to several medications, including antimicrobial agents such as amphotericin B, anti-inflammatory agents such as methotrexate, biologic agents such as bevacizumab, and cardiovascular agents and chemotherapeutic agents. We describe the case of a 73-year-old female who developed interstitial lung disease following chronic use of nitrofurantoin for a urinary tract infection (UTI). The patient was taking nitrofurantoin $100 \mathrm{mg}$ capsules twice daily for approximately 3 years. She presented to the hospital with complaints of a persistent dry cough that started 2 years previously. Her chest radiograph revealed bilateral reticular opacities and some atelectasis. Computed tomography of the chest demonstrated development of subpleural reticular opacities with minimal honeycombing. The patient had a severe restrictive defect on her pulmonary function tests, with a significant reduction in her carbon monoxide diffusion capacity. Multiple infectious disease and autoimmune tests were negative. Utilizing the algorithm of Naranjo (score of 9), it was determined that chronic use of nitrofurantoin was the definite cause of the patient's interstitial lung disease. Nitrofurantoin was discontinued and she was treated with oxygen and started on an oral steroid, both of which were continued permanently once discharged. Upon discharge, the patient was maintained on $5 \mathrm{~L}$ of oxygen at rest and $10 \mathrm{~L}$ of oxygen when ambulating. Unfortunately, her lung disease ultimately resulted in her demise several months
\end{abstract}

Charles F. Seifert

charles.seifert@ttuhsc.edu

1 School of Pharmacy, Texas Tech University Health Sciences Center, 3601 4th Street, STOP 8162, Lubbock, TX 79430-8162, USA after her diagnosis. This case report illustrates the importance of rapid recognition of drug-induced lung injuries and discontinuation of the offending agent.

\section{Key Points}

Chronic use of nitrofurantoin can lead to pulmonary complications such as interstitial lung disease.

Nitrofurantoin should be stopped upon the first sign of lung injury.

Nitrofurantoin should only be considered if other therapies have failed and suitable patient monitoring is available.

\section{Background}

Drug-induced interstitial lung disease is a rare condition attributed to several medications, including antimicrobial agents such as amphotericin B, anti-inflammatory agents such as methotrexate, biologic agents such as bevacizumab, and cardiovascular agents and chemotherapeutic agents [1]. The current Infectious Diseases Society of America and European Society of Microbiology and Infectious Diseases guidelines recommend nitrofurantoin as a first-line medication for the treatment of uncomplicated cystitis and suppression of recurrent urinary tract infections (UTIs), a common problem prevalent in females $[2,3]$. The classic presentation of interstitial lung disease usually occurs within 3-8 days of starting nitrofurantoin, 
but may occur a few hours to 4 weeks after the first dose [4]. Confirmatory diagnosis is based on clinical imaging and histopathology, documentation of drug exposure, improvement after discontinuing the agent, and symptom onset following a rechallenge with the offending agent $[5,6]$. We report a case of interstitial lung disease as a result of chronic exposure to nitrofurantoin, as well as a review of the current literature.

\section{Case Report}

In early 2015, a 73-year-old female presented for evaluation of a persistent dry cough that started 2 years ago and had progressively worsened with exertional dyspnea over the past week. Her hypertensive medication, lisinopril, was changed to valsartan several months ago, without any improvement in her cough. Her past medical history was remarkable for hypertension, chronic UTIs, hyperlipidemia, and hypothyroidism. She had recurrent UTIs and was taking nitrofurantoin $100 \mathrm{mg}$ twice daily for approximately 3 years preceding the onset of symptoms. She had no previous lung disease, smoking history, or past work exposure. On physical examination, the patient was afebrile and appeared mildly distressed, fatigued, and short of breath, with a non-productive cough. She denied any chest pain. Her respiratory rate was 20 per minute, and she had a blood pressure of $157 / 94 \mathrm{mmHg}$, pulse of 102 beats per minute, and a hemoglobin oxygen saturation of $90 \%$ on room air. Laboratory studies revealed a white blood cell count of $8.82 / \mathrm{mm}^{3}$ (range $4.3-10.60$ ), with no blood eosinophilia present. Her chemistry panel was within the normal range. The erythrocyte sedimentation rate was $18 \mathrm{~mm} / \mathrm{h}$ (normal range $0-29 \mathrm{~mm} / \mathrm{h}$ for women) and d-dimer was $<150 \mathrm{ng} /$ $\mathrm{mL}$. She was euthyroid, and enzyme-linked immunosorbent assay for human immunodeficiency virus was negative. Antinuclear antibody, antineutrophil cytoplasmic antibody, and rheumatoid factor were normal. The chest radiograph revealed bilateral reticular opacities and some atelectasis (Fig. 1), and computed tomography (CT) of the chest demonstrated development of subpleural reticular opacities with minimal honeycombing (Fig. 2). Pulmonary function tests showed a severe restrictive defect (forced vital capacity [FVC] $1.18 \mathrm{~L}, 36.6 \%$ of predicted, forced expiratory volume at $1 \mathrm{~s}[\mathrm{FEV} 1] / \mathrm{FVC}$ was $95.2 \%$, FEV1 $1.12 \mathrm{~L}, 45.2 \%$ of predicted, suggestive of restriction) with severely reduced carbon monoxide diffusion capacity (DLCO $17.3 \%$, diffusing capacity corrected for alveolar volume [DLCO/VA], $49.4 \%$ predicted). The total lung capacity (TLC) of $2.98 \mathrm{~L}$ was $53.5 \%$ of predicted and confirmed restriction, in addition to the residual volume (RV) being $1.80 \mathrm{~L}$, which was $78.9 \%$ of predicted. Overall, there was severely decreased lung capacity and decreased

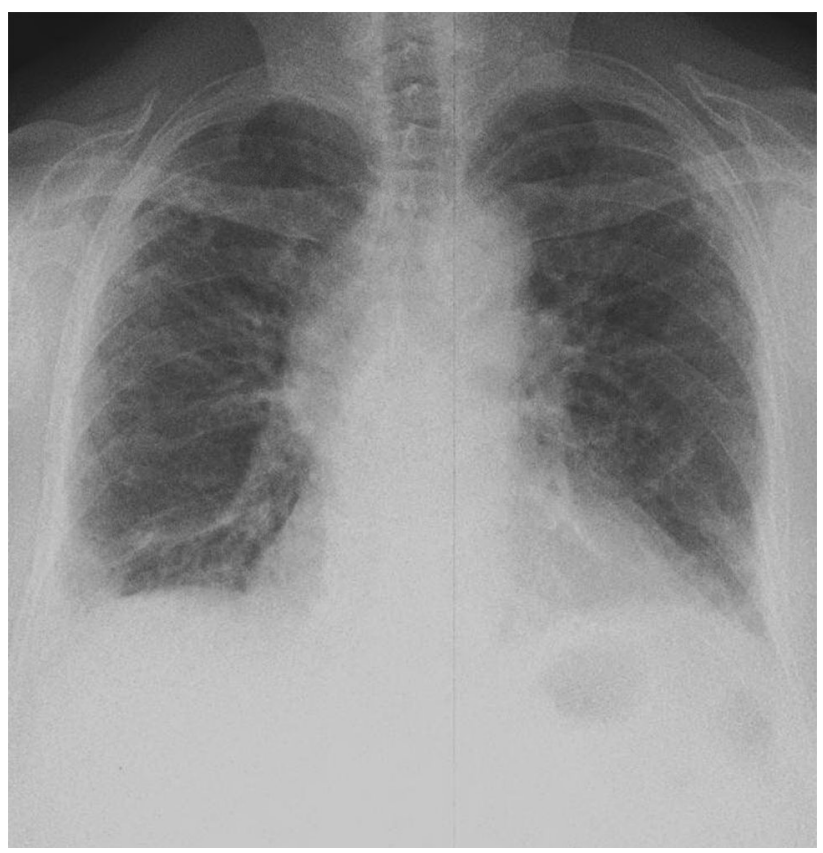

Fig. 1 Chest radiograph revealed bilateral reticular opacities and some atelectasis

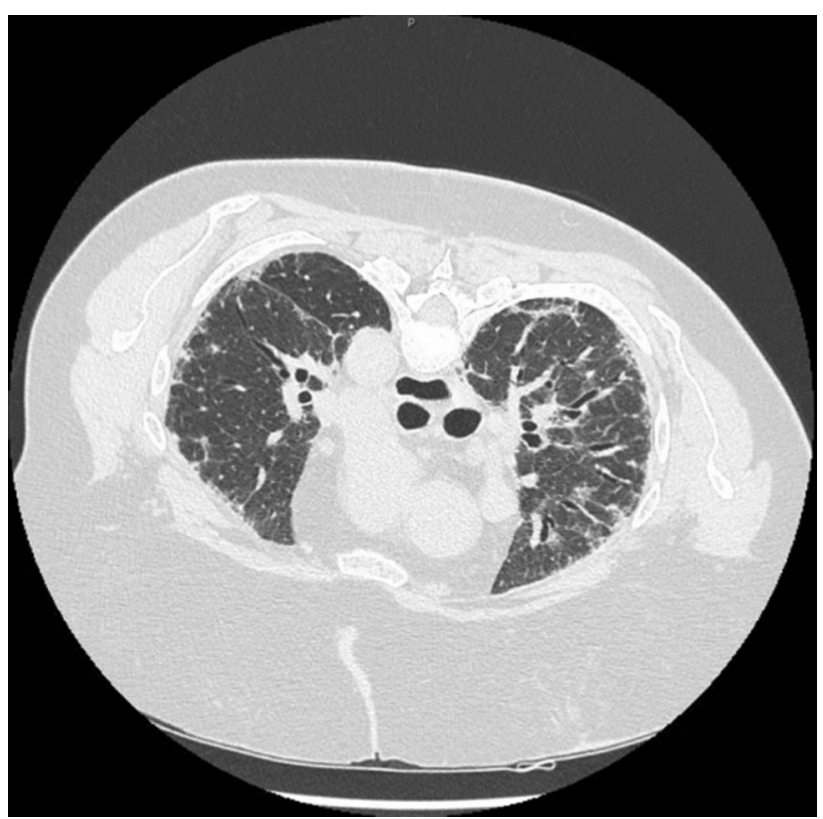

Fig. 2 High-resolution computed tomography of the chest in the prone position showing development of subpleural reticular opacities with minimal honeycombing

diffusion capacity. Electrocardiograph and echocardiography were normal, along with all other cardiology findings. Nitrofurantoin-induced lung disease was suspected with a differential diagnosis of pneumonia and pulmonary embolism. Empiric community-acquired pneumonia treatment was initiated with piperacillin-tazobactam $3.375 \mathrm{~g}$ intravenously every $6 \mathrm{~h}$, vancomycin $1.5 \mathrm{~g}$ intravenous 
piggy back every $12 \mathrm{~h}$, and methylprednisolone $40 \mathrm{mg}$ intravenously every $6 \mathrm{~h}$. The antibiotics were discontinued on day 2 . The day following admission (day 2), the patient still experienced shortness of breath, mainly with activity, and her oxygen saturation dropped to $50 \%$ while she was receiving $5 \mathrm{~L}$ of oxygen. She required $10 \mathrm{~L}$ of oxygen to maintain saturation above $85 \%$ while ambulating. Her cough was now productive, with scanty whitish sputum, and antibiotic therapy was de-escalated to levofloxacin $750 \mathrm{mg}$ daily as the patient was afebrile with a normal white blood cell count. Methylprednisolone was discontinued on day 3 and the patient was started on prednisone $60 \mathrm{mg}$ daily by mouth. Nitrofurantoin was discontinued. On day 3 , the patient utilized $5 \mathrm{~L}$ of oxygen while lying in bed and $10 \mathrm{~L}$ of oxygen when ambulating. She denied any fever or chest pain overnight. There was no blood culture growth and the sputum culture was indicative of normal upper respiratory flora. She was maintained on $5 \mathrm{~L}$ of oxygen at rest and $10 \mathrm{~L}$ of oxygen when ambulating, and was discharged the following day with 5 more days of levofloxacin therapy and a taper regimen for prednisone, such that she would be on a maintenance dose of $30 \mathrm{mg}$ daily after tapering. Her nitrofurantoin was switched to cephalexin $500 \mathrm{mg}$ four times daily for long-term prophylaxis. Months after, the patient became septic secondary to pneumonia, which lead to her demise.

\section{Discussion}

Since 1953, nitrofurantoin has been used for uncomplicated UTIs with a low resistance. Nitrofurantoin works by inhibiting aerobic energy metabolism, DNA synthesis, RNA synthesis, cell wall synthesis, and protein synthesis. It is active against gram-positive aerobes such as Staphylococcus saprophyticus, and gram-negative aerobes such as Escherichia coli [7]. The package insert for nitrofurantoin specifically warns about pulmonary reactions, including, but not limited to, diffuse interstitial pneumonitis and pulmonary fibrosis [8].

There is no consensus explanation or description pertaining to the mechanism of action of nitrofurantoin-induced interstitial lung disease. Hypersensitivity to nitrofurantoin is one of the proposed mechanisms of action and is thought to be the cause of acute presentation of lung damage [9-12]. It is theorized that the lymphocytes activated by nitrofurantoin facilitate the release of cytokines, which result in lymphocytic alveolitis, or more commonly known as extrinsic allergic alevolitis $[10,13]$. As this is a theorized hypersensitivity and inflammatory reaction, steroid therapy may be considered. Other theorized mechanisms of action include disruption in the balance of oxidants and antioxidants in the lung, as well as direct damage to the parenchymal cells of the lung. When the balance of oxidants and antioxidants are disrupted, toxic products are formed, which can cause interstitial lung fibrosis [9, 10, 14]. Direct toxicity may be correlated with dose and as such would be more applicable to the chronic presentation of lung damage; however, to our knowledge, there have not been any studies showing a direct correlation between dose of nitrofurantoin and interstitial lung disease.

The variable clinical presentation of nitrofurantoin-induced interstitial lung disease makes diagnosis difficult, and thus withdrawal of the drug is oftentimes delayed. Ideally, viral studies and tests for hypersensitivity pneumonitis should be performed; however, diagnosis of interstitial lung disease secondary to nitrofurantoin use has become more of a process of exclusion. Nitrofurantoin interstitial lung disease can be categorized as acute or chronic and, to date, has not been correlated with dose dependence [15]. Acute manifestation is more prevalent than chronic manifestation, and its presentation consists of a maculopapular rash, fever, fatigue, arthralgia, dyspnea, cough, and chest pain, and, in some cases pulmonary edema. Eosinophilia in the serum or bronchoalveolar lavage may also be present but is not indicative of an acute manifestation as it may also be present in chronic manifestation $[9,12]$. The onset of symptoms may occur within hours to 2 weeks upon starting the drug. With acute presentation, the pulmonary function test may show a restrictive flow with reduced diffusion capacity. The major difference between acute and chronic is that the symptoms will tend to dissipate in the former rather than the latter once nitrofurantoin is discontinued $[9,11,12]$.

Chronic presentation is infrequent and occurs in patients who have been utilizing nitrofurantoin for at least 6 months or more. It is interesting to note that prior acute reactions do not potentiate the development of a chronic reaction $[16,17]$. Chronic presentation occurs over weeks to years and consists of a progressive dry cough and progressive dyspnea [18-20]. With chronic presentation, clubbing, inspiratory crepitations, and bilateral interstitial infiltrates on a chest X-ray may be present. Additionally, a restrictive flow with reduced diffusion capacity may be seen on the pulmonary function test. Of note, in chronic presentation there is marked honeycombing of the lung(s) seen on high resolution CT scans $[19,20]$.

Our patient's respiratory symptoms could have been attributed to pulmonary congestion, indicating possible heart failure. In addition, her prior use of lisinopril could have been the cause of her cough; however, considering the patient had been taking these medications for years, they were ruled out as causative agents. Our patient presented with more of a chronic presentation as she had prior exposure to nitrofurantoin for more than 2 years.

There is a paucity of data to indicate gender as a risk factor for drug-induced interstitial lung disease; however, 
in the literature, patients of older age have been shown to be more susceptible to drug-induced interstitial lung disease. This may be attributed to the fact that as a person ages, their renal function declines, and blood flow to the liver and general circulation is decreased, in addition to slowed metabolism [1,21]. Our patient's calculated creatinine clearance was $50.7 \mathrm{~mL} / \mathrm{min}$ and, according to the product information for nitrofurantoin, use in creatinine clearance $<60 \mathrm{~mL} / \mathrm{min}$ is contraindicated [22].

Based on the Naranjo algorithm [23], our patient had a score of 9 , indicating the interstitial lung disease was definitely caused by nitrofurantoin. This diagnosis is further supported by clinical imaging, documentation of drug exposure, and mild improvement after discontinuing nitrofurantoin. Treatment begins with discontinuation of nitrofurantoin, which usually results in clinical improvement within $24 \mathrm{~h}$, with other findings such as chest radiograph opacities requiring a longer period of time to resolve. The literature suggests high-resolution CT is superior to chest radiography and is valuable in detecting interstitial lung disease when compared with the latter [6]. Depending on the clinical presentation of the patient, bronchodilators, oxygen, and short bursts of steroids can be utilized to abate symptoms [16, 24]. With respect to our patient, prednisone was utilized as an inpatient and then continued long-term as an outpatient.

With the rising resistance to fluoroquinolones and trimethoprim/sulfamethoxazole, nitrofurantoin is more commonly being considered for the treatment of recurrent UTIs. However, the link between the rare but serious adverse effect between nitrofurantoin and interstitial lung disease is well-documented in the literature [5, 25-29] and should be taken into account when selecting a chronic prophylactic regimen. Studies have shown nitrofurantoin to be clinically efficacious to ciprofloxacin, third-generation cephalosporins, and amoxicillin for the treatment of UTIs $[2,7,30]$. Thus, there are safer alternatives that can be considered and these should be tried prior to initiating a patient on nitrofurantoin, taking into consideration local institution resistance rates.

\section{Conclusions}

Nitrofurantoin-induced interstitial lung disease is a welldocumented phenomenon that often goes misdiagnosed due to its presentation, and is often treated as pneumonia, myocardial infarction, heart failure, and even pulmonary embolism. It is important to realize the adverse effects of nitrofurantoin and their clinical presentation, and carefully evaluate the risk versus benefit prior to initiating treatment. Patients receiving nitrofurantoin long-term should be assessed periodically. In the event a patient is prescribed nitrofurantoin, the pharmacist plays an integral role in counseling patients and stressing the importance of reporting breathing difficulty or unusual symptoms to their prescriber as soon as possible.

\section{Compliance with Ethical Standards}

Conflict of interest Kerry Anne Rambaran and Charles F. Seifert declare that they have no conflicts of interest.

Consent Written informed consent was obtained from the patient, prior to her death, for publication of this case report and any accompanying images. A copy of the written consent may be requested for review from the corresponding author.

Funding No funding was received for the publication of this case report.

Open Access This article is distributed under the terms of the Creative Commons Attribution-NonCommercial 4.0 International License (http://creativecommons.org/licenses/by-nc/4.0/), which permits any noncommercial use, distribution, and reproduction in any medium, provided you give appropriate credit to the original author(s) and the source, provide a link to the Creative Commons license, and indicate if changes were made.

\section{References}

1. Schwaiblmair M, Behr W, Haeckel T, Märkl B, Foerg W, Berghaus T. Drug induced interstitial lung disease. Open Respir Med J. 2012;6:63-74

2. Gupta K, Hooton TM, Naber KG, Wullt B, Colgan R, Miller LG, et al. Executive summary: international clinical practice guidelines for the treatment of acute uncomplicated cystitis and pyelonephritis in women: a 2010 update by the Infectious Diseases Society of America and the European Society for Microbiology and Infection. Clin Infect Dis. 2011;52(5):561-4.

3. Lichtenberger P, Hooton TM. Antimicrobial prophylaxis in women with recurrent urinary tract infections. Int $\mathbf{J}$ Antimicrob Agents. 2011;38(Suppl):36-41.

4. Kabbara WK, Kordahi MC. Nitrofurantoin-induced pulmonary toxicity: a case report and review of the literature. J Infect Public Health. 2015;8(4):309-13.

5. Camus P, Bonniaud P, Fanton A, Camus C, Baudaun N, Foucher $\mathrm{P}$. Drug-induced and iatrogenic infiltrative lung disease. Clin Chest Med. 2004;25(3):479-519, vi.

6. Bradley B, Branley HM, Egan JJ, Greaves MS, Hansell DM, Harrison NL, et al. Interstitial lung disease guideline: the British Thoracic Society in collaboration with the Thoracic Society of Australia and New Zealand and the Irish Thoracic Society. Thorax. 2008;63(Suppl 5):v1-58.

7. Huttner A, Verhaegh EM, Harbarth S, Muller AE, Theuretzbacher U, Mouton JW. Nitrofurantoin revisited: a systematic review and meta-analysis of controlled trials. J Antimicrob Chemother. 2015;70(9):2456-64.

8. Macrobid (nitrofurantoin) package insert 2009. Cincinnati, OH: Proctor and Gamble Pharmaceuticals.

9. Hainer BL, White AA. Nitrofurantoin pulmonary toxicity. J Fam Pract. 1981;13(6):817-23.

10. Goemaere NNT, Grijm K, van Hal PTW, den Bakker MA. Nitrofurantoin-induced pulmonary fibrosis: a case report. J Med Case Rep. 2008;2:169.

11. Boggess KA, Benedetti TJ, Raghu G. Nitrofurantoin-induced pulmonary toxicity during pregnancy: a report of a case and 
review of the literature. Obstet Gynecol Surv. 1996;51(6):367-70.

12. Witten CM. Pulmonary toxicity of nitrofurantoin. Arch Phys Med Rehabil. 1989;70(1):55-7.

13. Simonian SJ, Kroeker EJ, Boyd DP. Chronic interstitial pneumonitis with fibrosis after long-term therapy with nitrofurantoin. Ann Thorac Surg. 1977;24(3):284-8.

14. Boyd MR, Catignani GL, Sasame HA, Mitchell JR, Stiko AW. Acute pulmonary injury in rats by nitrofurantoin and modification by vitamin E, dietary fat, and oxygen. Am Rev Respir Dis. 1979;120(1):93-9.

15. D'Arcy PF. Nitrofurantoin. Drug Intell Clin Pharm. 1985;19(7-8):540-547.

16. Holmberg L, Boman G, Böttiger LE, Eriksson B, Spross R, Wessling A. Adverse reactions to nitrofurantoin. Analysis of 921 reports. Am J Med. 1980;69(5):733-8.

17. Bhullar S, Lele SM, Kraman S. Severe nitrofurantoin lung disease resolving without the use of steroids. J Postgrad Med 2007;53(2):111-113.

18. Vahid B, Wildemore B. Nitrofurantoin pulmonary toxicity: a brief review. Internet J Pulm Med. 2005;6(2). http://ispub.com/ IJPM/6/2/11191. Accessed 10 May 2016.

19. Mendez JL, Nadrous HF, Hartman TE, Ryu JH. Chronic nitrofurantoin-induced lung disease. Mayo Clin Proc. 2005;80(10):1298-302.

20. Martins RR, Marchiori E, Viana SL, Grillo Júnior LSP, Capelozzi VL, Valença LM. Chronic eosinophilic pneumonia secondary to long-term use of nitrofurantoin: high-resolution computed tomography findings. J Bras Pneumol. 2008;34(3):181-4.

21. Simpson AB, Paul J, Graham J, Kaye SB. Fatal bleomycin pulmonary toxicity in the west of Scotland 1991-95: a review of patients with germ cell tumours. $\mathrm{Br} \mathrm{J}$ Cancer. 1998;78(8):1061-6.

22. Product information: Macrodantin ${ }^{\circledR}$ oral capsules, nitrofurantoin macrocrystals oral capsules. NJ: Almatica Pharma, Inc. (per FDA); Pine Brook.

23. Naranjo CA, Busto U, Sellers EM, et al. A method for estimating the probability of adverse drug reactions. Clin Pharmacol Ther. 1981;30(2):239-45.

24. Holmberg L, Boman G. Pulmonary reactions to nitrofurantoin. 447 cases reported to the Swedish Adverse Drug Reaction Committee 1966-1976. Eur J Respir Dis. 1981;62(3):180-9.

25. Kanji Z, Su VCH, Mainra R. Nitrofurantoin-induced pulmonary reaction involving respiratory symptoms: case report. Can J Hosp Pharm. 2011;64(5):362-5.

26. Lee B, Balavenkataraman A, Sanghavi D, Walter K. Recurrent nitrofurantoin-induced giant cell interstitial pneumonia: Case report and literature review. Respir Med Case Reports. 2015;14:49-52.

27. Madani Y, Mann B. Nitrofurantoin-induced lung disease and prophylaxis of urinary tract infections. Prim Care Respir J. 2012;21(3):337-41.

28. Naidu GD, Deepthi P, Karthik KR, Das U, Swarnalatha G, Gangadhar T. Nitrofurantoin induced interstitial lung disease. Indian J Nephrol. 2014;24(6):405.

29. Syed H, Bachuwa G, Upadhaya S, Abed F. Nitrofurantoin-induced interstitial pneumonitis: albeit rare, should not be missed. BMJ Case Rep. 2016. doi:10.1136/bcr-2015-213967

30. Jancel T, Dudas V. Management of uncomplicated urinary tract infections. West J Med. 2002;176(1):51-5. 